\title{
II COLOQUIO HISPANO-MARROQUI'DE CIENCIAS HISTÓRICAS «HISTORIA, CIENCIA Y SOCIEDAD», GRANADA, 6-11 DE NOVIEMBRE DE 1989
}

Por

FRANCISCO FRANCO SÁNCHEZ

Entre los encuentros científicos internacionales recientes hay que destacar el celebrado entre los días 6 al 11 de noviembre de 1989 en Granada, el // Coloquio Hispano-Marroqui de Ciencias Históricas «Historia, Ciencia y Sociedad». Organizado por el Instituto de Cooperación con el Mundo Árabe del Ministerio de Asuntos Exteriores, el Ministerio de Asuntos Culturales de Rabat y la Escuela de Estudios Árabes de Granada, tuvo sus sesiones científicas en la Casa del Chapiz del C.S.I.C., en Granada.

Se presentaron un apreciable número de ponencias, en árabe y español, tanto por parte de los investigadores marroquíes, como por los españoles, siendo destacable el alto nivel científico del Coloquio. El lunes 6 de noviembre por la mañana tuvo lugar en el Rectorado de la Universidad de Granada su inauguración, asistiendo a la misma Luis Yáñez (Secretario de Estado de Cooperación Internacional y para Iberoamérica y Presidente de la Comisión Nacional del V Centenario y de «Al-Andalus-92»), Luis Peset (del Instituto de Ciencias Históricas, representando al Presidente del Consejo Superior de Investigaciones Científicas), Gerardo Entrena Cuesta (Gobernador Civil de Granada), Antonio Caro (Alcalde de Granada), Pascual Ribas (Rector de la Universidad de Granada); todos ellos hablaron en nombre de las entidades que representaban, haciendo ver lo positivo de estos intercambios culturales.

Por la tarde, en la Escuela de Estudios Árabes del C.S.I.C., situada en la Casa del Chapiz, tras la presentación y recepción oficial por Luis Molina (Director de la misma) se dio comienzo a las sesiones científicas, con la exposición de las siguientes ponencias: Joaquín VALLVÉ BERMEJO (Real Academia de la Historia de Madrid): "Etnografía y toponimia»; Mohammed BENABOUD (Univ. de Tetuán): "Estructuras sociales en Al-Andalus y en el Mágreb: El tribalismo»; Pedro CHALMETA GENDRÓN 
(Univ. de Zaragoza): "La conquista del 711-713 y la formación de Al-Anda/us"; Juan CASTILLA BRAZALES (C.S.I.C. de Granada): «Crónicas magrebies para la historia de Al-Andalus», y la de Mohammed HAJJI (Univ. de Rabat): "La escuela maliki en Al-Andalus y en el Mágreb".

En las sesiones matinales del martes 7 (jornada dedicada a la historia y sociedad en general) se expusieron las ponencias de: Jorge LIROLA DELGADO (Univ. de Granada): «Reflexiones sobre el poderío naval en el Occidente musulmán (VIII-X). Fuentes para su estudion; Juan Antonio SOUTO LASALA (Univ. Complutense de Madrid): «Fuentes magrebies sobre la Marca Superior de Al-Andalus. El volumen // de Al-Bayân al-Mugrib de Ibn 'Idârì); M. ${ }^{a}$ Jesús VIGUERA MOLINS (Univ. Complutense de Madrid): «Relaciones entre el Mágreb y Al-Andalus en el siglo Xl»; Victoria AGUILAR SEBASTIÁN (C.S.I.C. Madrid): «Politica de 'Abd Al-Mu'min con las tribus árabes de Ifriqiya»; Angel C. LÓPEZ y Antonio ORIHUELA (C.S.I.C. de Granada): "Una nueva interpretación del texto de Aben Al-Jatib sobre la Alhambra en 1362"; Francisco VIDAL CASTRO (Univ. de Granada): «La interacción jurídica, social y económica entre Al-Andalus y el Mágreb a través del Mi'yār de Al-Wanšărisis's; Mohammed RAZZOUK (Univ. de Casablanca): "La experiencia morisca en Marruecos en los siglos $X V / /$ Y XV/l/s.

Por la tarde hablaron Elena DE FELIPE RODRÍGUEZ (C.S.I.C. de Madrid): «Familias de ulemas de origen beréber en Al-Anda/US»; Rafael VALENCIA RODRIGUEZ (Univ. de Sevilla): "La emigración sevillana hacia el Mágreb alrededor de 1248»; Ismat DANDASH (Univ. de Rabat): «El papel politico de la mujer almorávide» y, por último, Francisco FRANCO SÁNCHEZ (Univ. de Alicante): "Andalusies y magrebies en torno a los Sid Bonola de Guadalest y Granada", recogiendo e interpretando las noticias conocidas de esta familia de místicos originaria de Guadalest (Alicante), trasladada a Granada y luego convertidos al cristianismo tras la conquista.

El miércoles 8 , las ponencias estuvieron centradas en la historia de la ciencia árabe, así, por la mañana, Mercè COMES (Univ. de Barcelona) inició las exposiciones con su disertación «En torno a la influencia de Azarquiel en el Norte de África. El apogeo solar y la oblicuidad de la eclíptica en el ziỳ de Ibn Ishäq", ponencia seguida por la de Miquel FORCADA (Univ. de Barcelona): «Fuentes andalusies del Calendario de Ibn al-Bannā' de Marrākuš»;; Ingrid BEJARANO (Univ. de Barcelona): «Referencia histórica, geográfica y cientifica sobre el Mágreb en la obra cosmográfica del viajero andalusi Abū Hāmid al-Garnățis; Mohamed MANOUNI (Univ. de Rabat): «Relaciones cientificas entre el Mágreb y Al-Andalus en la Edad Media»; Margarita CASTELLS (Univ. de Barcelona): «Médicos andalusies en Marruecos en los siglos $X I I I-X V$ » y Braulio JUSTEL CALABOZO (Univ. de Cádiz): «El facultativo Coll en la corte de Muley Solimán y su Diccionario elemental de fármacos»; e Ildelfondo GARIJO GALÁN (Univ. de Córdoba): «Acerca de algunos tratados sobre la tríaca en el Mágreb». Por la tarde se dio la oportunidad de cursar una visita al barrio granadino del Albaicín. Guiados por los anfitriones granadinos, los asistentes al Coloquio pudieron. ver los restos del Bañuelo, del Maristán y por la noche fueron conducidos al Palacio de Córdoba.

El jueves 9 de noviembre estuvo dedicado preferentemente al derecho musul-

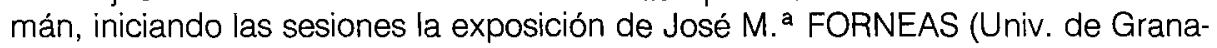


da): "El Kitäb al-Muwatta' de Mälik B. Anas en Al-Andalus. Datos para el estudio de su transmisión y difusión» y siguiendo las de Ahmed TORRISI (Univ. de Rabat): «lbn Al-Jatib: una manifestación poética andalusil/magrebi excepcional; M. ${ }^{\text {a }}$ ARCAS CAMPOY (Univ. de La Laguna): "Evolución y valoración actual de la literatura jurídica»; M. ${ }^{a}$ José CERVERA (Univ. de Zaragoza): "El Muhtasar de Al-Tulayțti (siglo X). Primer compendio jurídico andalusi conservado"; Alfonso CARMONA GONZÁLEZ (Univ. de Murcia): "El Madhab juridico-religioso del Occidente musulmán visto por geógrafos y viajeros orientales"; finalizando la sesión matinal Mohammed KETTANI (Univ. de Tetuán): "La escuela maliki en el Mágreb y Al-Andalus".

En la asamblea vespertina intervinieron Pedro CANO ÁVILA (Univ. de Sevilla): «El notario musulmán andalusi»; Hassan WARAGLI (Univ. de Tetuán): "La escuela malikien Al-Andalus y el Mágreb»; Rafael PINILLA (Univ. de Córdoba): «Unas fatwas atribuidas a Ibn Luyūn». Tras la sesiones científicas, se hizo una visita nocturna a la Alhambra.

El viernes 10 de noviembre por la mañana Mohamed BENCHERIFA (Real Academia de Marruecos, Rabat) abrió las sesiones con su disertación sobre «Una personalidad de finales de la época granadina: Al-Mintawris, continuando Celia del MORAL (Univ. de Granada): Poetas granadinos del Norte de África"; Marcelino VILLEGAS (Univ. de Alicante) sobre «España y el Mágreb en la obra de Sarmiento», en que analizaba la visión y el pensamiento de este escritor sudamericano tras sus viajes por España y el Mágreb a fines del pasado siglo; Aziza BENNANI (Univ. de Mohammedia): «Relaciones hispano-marroquies en el siglo XIX»; Ahmed Chauki BINBIN (Univ. de Rabat): «Las relaciones hispano-marroquies después de la apropiación de la Biblioteca Zaydani, actualmente en El Escorials; Hossain BUZINEB (Univ. de Rabat): "La cesión de la Alcazaba de Salé-Siglo XVIIl".

Por la tarde se expusieron las ponencias de Mohamed ZNIEBER (Univ. de Rabat): «Influencia de la cultura andalusi en el desarrollo cientifico de ambos países»; Rafael MUÑNZ JIMÉNEZ (Univ. de La Laguna): «Una metáfora reveladora para entender a lbn Jaldün", y cerrando este Coloquio la de Abdallah LAROUI (Real Academia de Marruecos, Rabat): «Pensamiento histórico e historicismo».

Todas las sesiones científicas tuvieron al final de las mismas animados debates, que contribuyeron a hacer más interesantes las aportaciones reseñadas. La clausura tuvo lugar en la misma Casa de Chapiz; intervinieron sucesivamente Luis Molina (representando a la Escuela de Estudios Árabes de Granada), Abdelhakim Bsiri (del Ministerio de Asuntos Culturales de Rabat), José Pérez Lázaro (del Instituto para la Cooperación con el Mundo Árabe de Madrid) y Almudena Tarrasa (Sub-directora de esta última institución). Seguidamente se leyó un comunicado conjunto en que se exhortaba a la cooperación cultural, científica e investigadora entre España y Marruecos y se proponian unos cauces para la misma. Finalmente, hemos de resaltar la excelente labor de los organizadores del Coloquio y la buena acogida de las instituciones granadinas, especialmente de la Casa del Chapiz. Esperemos que la pronta publicación de las Actas del Congreso sea el colofón a una reunión científica tan positiva. 